\title{
Improvement of generation change on SSE algorithm
}

\author{
T. Maruyama \& E. Kita \\ Graduate School of Information Sciences, \\ Nagoya University, Nagoya, Japan
}

\begin{abstract}
The Stochastic Schemata Exploiter (SSE) is one of the evolutionary optimization algorithms for solving the combinatorial optimization problems. For improving the search performance of SSE, we will present the cross generational elitist selection SSE (cSSE) algorithms of which the generation alternation algorithm is improved by using cross generational elitist selection. The SSE and the cSSE are compared with the Minimal Generation Gap (MGG) and the Bayesian Optimization Algorithm (BOA) on deception and knapsack problems in order to discuss their features.
\end{abstract}

\section{Introduction}

The actual industrial problems are often formulated as the multi-objective optimization problems defined with many design variables. Since it is very timeconsuming to find the solutions exactly, we have to obtain the quasi-optimum solutions in an admissible time. For this purpose, the evolutionary computations (ECs) are very useful tool [1-4].

Stochastic Schemata Exploiter (SSE) was presented by Aizawa [5] in 1994. The SSE is one of the EC and effective for the 0/1 combinational optimization problems. In the Genetic Algorithm (GAs), the individuals are generated randomly and the new ones are generated by the genetic operations such as the mutation, the crossover, the selection and so on. In the SSE, the other operations than the mutation are not necessary. Therefore, the total number of the control parameter of the SSE is much smaller than that of original GAs. Sub-populations are generated from the whole population according to the fitness ranking of the individuals. Schemata are extracted from the sub-population. New individuals are generated 
from the schemata. Since, in the SSE, the better schemata are distributed rapidly over the whole population, the convergence speed of the SSE is higher than that of the simple GAs. Once, however, the local optimum solution is found, it is difficult to find the other optimum solutions. Overcoming this difficulty, this paper presented Cross generational elitist selection SSE (cSSE), in which the cross generational elitist selection is introduced to the generation alternation of the SSE. The use of the cross generational elitist selection is to delete the identical individuals from the population and therefore, to improve the diversity of the population. In this paper, the cSSE is compared with the SSE, Minimal Generation Gap (MGG) and Bayesian Optimization Algorithm (BOA) on the deception and the knapsack problems.

The remainder of this paper is organized as follows. In section 2, Minimum Generation Gap (MGG), Bayesian Optimization Algorithm (BOA), Stochastic Schemata Exploiter (SSE), and cross-generational elitist selection SSE (cSSE) are introduced briefly. In section 3, the algorithms of the SSE and the cSSE are explained in more detailed. Numerical results are shown in section 4. In section 5, the conclusions are summarized.

\section{Background}

\subsection{Minimum Generation Gap (MGG)}

Genetic algorithm (GAs), which was presented by J. Holland in 1970s [1], is the optimization algorithm imitating the evolution process of the living organisms. After that, a lot of improved algorithm are developed from the GAs by many researchers.

Minimum Generation Gap (MGG) is one of the improved GAs [6]. The original GA has two difficulties; early convergence and evolutionary stagnation [3, 4]. In the MGG, the generation alternation process is improved for improving the search performance of the original GAs. The MGG is successfully applied to the actual problems such as the lens design, the protein structure analysis and so on $[7,8]$.

\subsection{Bayesian Optimization Algorithm (BOA)}

Estimation of Distribution Algorithm (EDA) is also very related to the GAs. In the EDA, the best solution is predicted according to stochastic model from the information of the better solutions in the population. The characteristic feature of the EDA is to predict the best solution by the distribution of better solutions in the population according to the stochastic model, instead of the evolutionary operators such as the crossover, mutation and so on.

The Bayesian Optimization AlgorithmiBOAjis one of the EDA, which is presented by Pelikan et al. [9]. In the BOA, the Bayesian network is used as the stochastic model to predict the best solution. 


\subsection{Stochastic Schemata Exploiter (SSE)}

Stochastic Schemata Exploiter (SSE), which was presented by Aizawa [5] in 1994, is one of the evolutionary algorithms.

It has very fast convergence property and small number of control parameters. While the original GA has a lot of control parameters such as population size, mutation rate, crossover rate and so on, the control parameters of SSE are only population size and mutation rate. In SSE, better schemata are extracted from the subpopulation of the better individuals and then, the new individuals are generated from the schemata. Since better schemata are spread over the population, the SSE has much better convergence property than the original GA. However, once it find a local optimum solution, it is difficult to find better solution.

\subsection{Cross-generational elitist selection SSE (cSSE)}

Since, in SSE, better schemata are distributed rapidly over the population, the SSE has better convergence property than the original GA. Instead of that, the diversity of individuals in the population is also lost rapidly in the relatively primary generation.

In cross-generational elitist selection SSE (cSSE), the generation alternation of the original SSE is improved by introducing cross-generational elitist selection of $\mathrm{CHC}[10]$. As a result, the diversity of the individuals in original SSE is enhanced.

\section{SSE and cSSE algorithms}

\subsection{SSE algorithm}

The key-point of SSE algorithm is not to select better individuals but to extract better schemata from the population. Since, it is difficult or very time-consuming to extract only better schemata from the population, the better schemata are extracted from sub-population composed of better individuals.

The population $P_{t}$ is made of $N$ individuals and the indices $c_{1}, c_{2}, \cdots, c_{N}$ mean the individuals re-arranged in descending order of their fitness. The sub-population arbitrarily selected from the population $P_{t}$ is referred to $S(\neq \phi)$, and the maximum index is to $L(S)$. If $L(S)<N$, the following partial order relationship is held among the sub-populations of the population $P_{t}$.

- The average fitness value of the sub-population $S$ is better than that of $S \cup c_{(L(S)+1)}$.

- The average fitness value of the sub-population $S$ is better than that of $\left(S-c_{L(S)}\right) \cup c_{(L(S)+1)}$.

where the symbol $\cup$ denotes the union of sets, and the population $\left(S-c_{k}\right)$ denotes the sub-population of the population $S$ in which the individual $c_{k}$ is taken away from the population $S$. 
In the SSE, the sub-populations are generated from the population $S=\left\{c_{1}\right\}$ according to the above partial order relationship. The algorithm of the SSE is as follows.

1. Individuals are sorted in descending order of their fitness in order to define the indices.

2. Sub-populations are generated according to the partial order relationship.

3. Common schemata are extracted from the sub-populations.

4. New individuals are generated randomly from the schemata.

5. The mutation operation is applied to the new individuals.

6. New population is constructed by the new individuals.

\section{2 cSSE algorithm}

If, in cSSE, the identical individuals are in the population, only one individual is left in the new population. For this purpose, cross generational elitist selection is employed. The cross generational elitist selection was presented by Eschelman in CHC [10].

The cSSE algorithm is as follows.

1. $N$ individuals are generated randomly to construct an initial population.

2. The individuals are sorted in descending order to make the list.

3. According to the SSE, $N$ new individuals are generated.

4. If there are the identical individuals in the population composed of $N$ old and $N$ new individuals, only one individual is left and the others are deleted.

5. New population is constructed by $N$ better individuals.

\section{Numerical example}

\subsection{Deception problem}

We will take as a first example, a deception problem [11]. The deception problem is defined as the summation of the small 4-bit deception problem defined as follows.

$$
\begin{array}{lll}
f_{d}(1111)=30 & f_{d}(0000)=28 & f_{d}(0001)=26 \\
f_{d}(0010)=24 & f_{d}(0100)=22 & f_{d}(1000)=20 \\
f_{d}(0011)=18 & f_{d}(0101)=16 & f_{d}(0110)=14 \\
f_{d}(1001)=12 & f_{d}(1010)=10 & f_{d}(1100)=8 \\
f_{d}(1110)=6 & f_{d}(1101)=4 & f_{d}(1011)=2 \\
f_{d}(0111)=0 . & &
\end{array}
$$

The objective function of the deception problem is defined as

$$
f_{\text {deception }}=\sum_{i=1}^{n} f_{d}\left(x_{i}\right),
$$




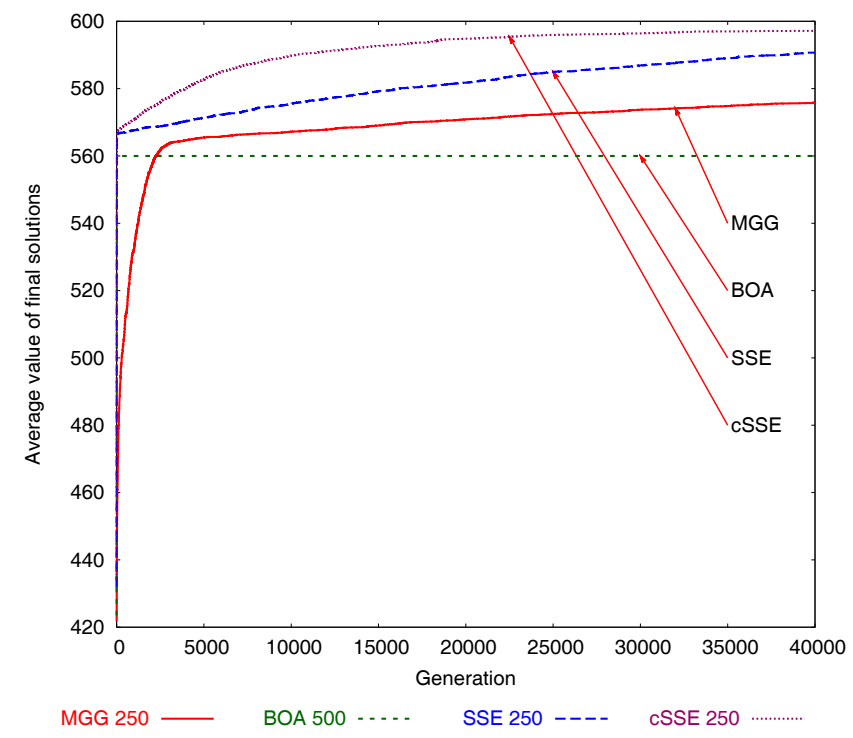

Figure 1: Average values of solutions on deception problem.

where $n=20$ denotes the total number of the small deception problems. The design variable $x_{i}$ is represented in 4-bit binary number;

$$
x_{i} \in 0000,0001, \cdots, 1111 \text {. }
$$

In MGG simulation, a two-point crossover with the crossover rate 1 is applied and all individuals are replaced at each generation. In the BOA simulation, half of all individuals are replaced at each generation. In the MGG, SSE and cSSE simulations, the best mutation rates are determined from some numerical experiments.

Maximum number of generations is 40000. Simulations are performed 50 times from different initial populations. Besides, the average and the standard deviation values of the fitness of the best individuals at each generation are illustrated in Figs. 1 and 2, respectively. The abscissa and the ordinate denote the generation step and the average and the standard deviation values of the fitness. Figure 1 illustrates that the convergence speed of MGG is the slowest and the cSSE is the fastest among them. Although BOA has fast convergence property at primary generation step, it is difficult to reach global minimum once it converges to the local minimum.

\subsubsection{Knapsack problem}

We shall consider as a second example a knapsack problem. A knapsack problem is a problem in combinatorial optimization. It derives its name from the maximization problem of choosing as much as possible essentials that can 


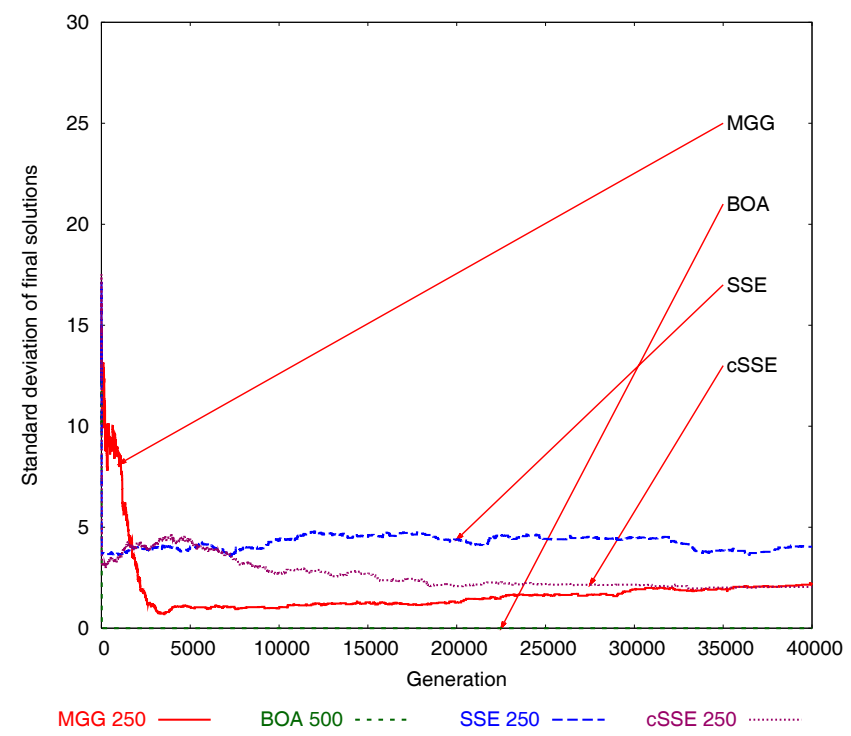

Figure 2: Standard deviation of solutions on deception problem.

fit into one bag (of maximum weight) you are going to carry on a trip. In the knapsack problem, baggage are selected so as to maximize their total amount on the condition that their total weight dose not overtake the previously defined limit;

$$
\begin{aligned}
& \max _{\left\{x_{i}\right\}} \sum_{i=1}^{n} c_{i} x_{i} \\
& \quad \text { subject to } \sum_{i=1}^{n} a_{i} x_{i} \leq b \\
& x_{i} \in 0,1 \quad(i=1, \cdots, n)
\end{aligned}
$$

where the baggage weight $a_{i}$ and the baggage amount $c_{i}$ are selected randomly within 1 to 100 . The weight limit is $b=10000$ and the total number of baggage is $n=400$.

The average and the standard deviation values of the fitness of the best individuals are illustrated in Figs. 3 and 4, respectively.

Figure 4 illustrates that the convergence speed of the standard deviation value of MGG is the slowest among them.

\section{Conclusions}

In this study, we presented the cross generational elitist selection SSE (cSSE) in which the generation alternation model of original SSE was replaced to cross 
Data Mining VII: Data, Text and Web Mining and their Business Applications 457

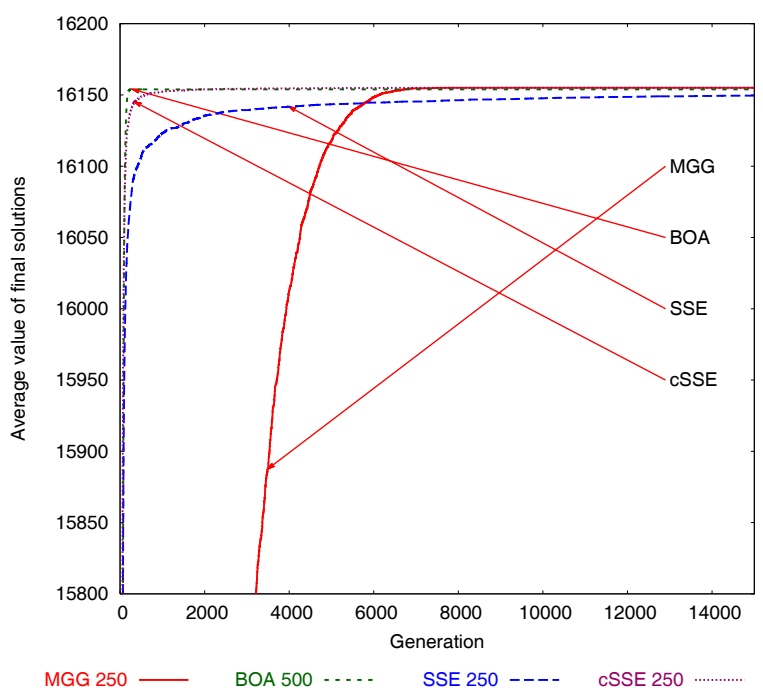

Figure 3: Average values of solutions on knapsack problem.

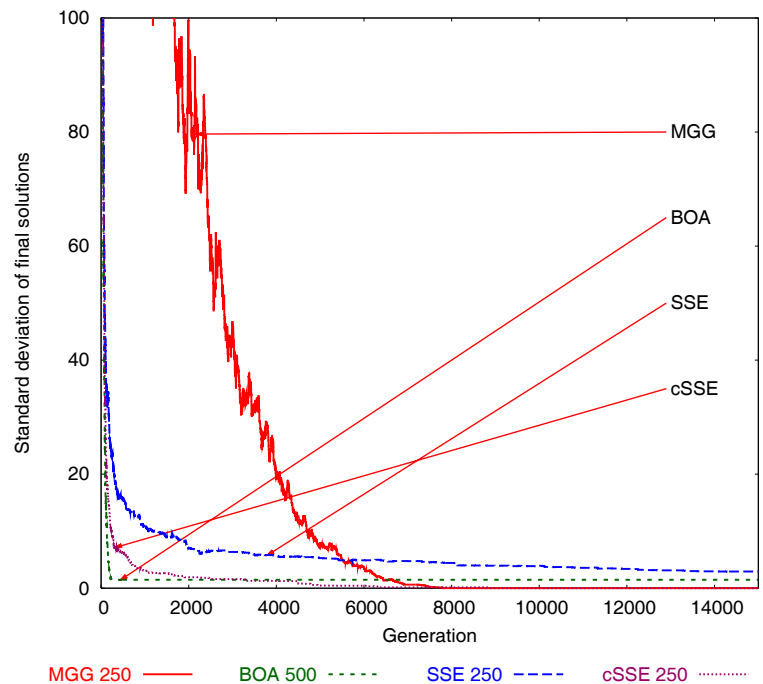

Figure 4: Standard deviation of solutions on knapsack problem.

generational elitist selection model of $\mathrm{CHC}$. The presented algorithm is compared with SSE, MGG and BOA.

We notice from the numerical examples the following features of the algorithms.

MGG has slower convergence speed than the others. However, it can find better solution than the others if the computational time is sufficient. 
The interesting features of BOA are very fast convergence speed at the primary step and low dependency of its search performance for the initially assumed population.

SSE, like BOA, has the low dependency of its search performance for the initially assumed population. Moreover, it can find better solution than that by the BOA.

cSSE has fast convergence speed at the primary step as the SSE. Moreover, it can find better final solution than the BOA and the SSE.

\section{References}

[1] J. H. Holland. Adaptation in Natural and Artificial Systems. The University of Michigan Press, 1 edition, 1975.

[2] L. Davis. Handbook of Genetic Algorithms. Van Nostrand Reinhold, 1 edition, 1991.

[3] J. E. Baker. Reducing bias and inefficiency in the selection algorithm. Proceedings of the Second International Conference on Genetic Algorithms, pp. 14-21, 1987.

[4] D. Whitley. The genitor algorithm and selection pressure: Why rankbased allocation of reproductive trials is best. Proceedings of the Third International Conference of Genetic Algorithms, pp. 116-121, 1989.

[5] N. Aizawa. Evolving SSE: A stochastic schemata explointer. In Proc. 1st IEE Conf. Evol. Comp., pp. 525-529. IEEE, 1994.

[6] H. Satoh, I. Ono, and S. Kobayashi. Minimal generation gap model for gas considering both exploration and exploitation. Proc. IIZUKA'96, pp. 494497, 1996.

[7] I. Ono, S. Kobayashi, and K. Yoshida. Global and multi-objective optimization for lens design by real-coded genetic algorithms. International Optical Design Conference, Vol. 3482, pp.110-121, 1998.

[8] O. Takahashi, H. Kita, and S. Kobayashi. Protein folding by a hierarchical genetic algorithm. The 4th International Symposium on Artificial Life and Robotics, pp. 334-339, 1999.

[9] M. Pelikan, D. E. Goldberg, and E. Cantu-Paz. Boa: The bayesian optimization algorithm. In W. Banzhaf, Daida J, A. E. Elben, M. H. Garzon, V. Honavar, M. Jakiela, and R. E. Smith, editors, Proceedings of the Genetic and Evolutionary Computation Conference 1999 (GECCO-1999, San Fransisco, CA), pp. 525-532. Morgan Kaufmann., 1999.

[10] L. J. Eshelman. The CHC adaptive search algorithm: How to have safe search when engaging in nontraditional genetic recombination. In G. J. E. Rawlins, editor, Foundations of Genetic Algorithms 1991 (FOGA 1), pp. 265-283. Morgan Kaufmann., 1991.

[11] L. D. Whitley. Fundamental principles of deception in genetic search. In G. J. E. Rawlins, editor, Foundations of Genetic Algorithms 1991 (FOGA 1), pp. 221-241. Morgan Kaufmann, 1991. 